Jurnal

\title{
ANALYSE DE L'EXPRESSION DE SENTIMENT DANS LE ROMAN UN SENTIMENT PLUS FORT QUE LA PEUR
}

Disusun dan Diajukan Oleh :

RAMAWATI LASTARIDA PURBA

2113131036

Telah diverifikasi dan Dinyatakan Memenuhi Syarat Untuk Diunggah Pada jurnal Online

Medan, November 2017

Menyetujui,

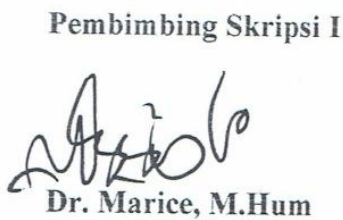

NIP. 196311201992032003

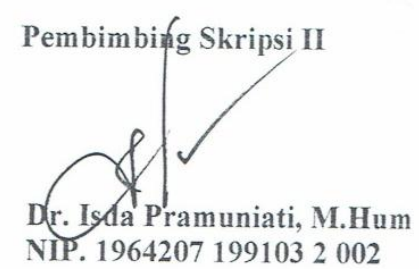

Editor Jurnal

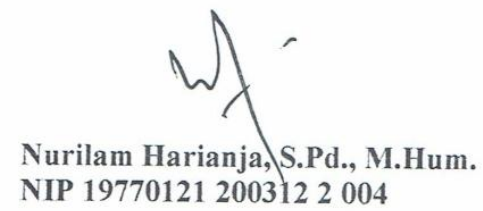




\title{
ANALYSE DE L'EXPRESSION DE SENTIMENT DANS LE ROMAN UN SENTIMENT PLUS FORT QUE LA PEUR
}

\author{
RAMAWATI LASTARIDA PURBA \\ Dr. Marice, M.Hum \\ Dr. Isda Pramuniati, M.Hum
}

\begin{abstract}
RÉSUMÉ
Ramawati Lastarida Purba, 2113131036. Analyse de l'expression de sentiments dans le roman Un Sentiment Plus Fort Que La Peur. Mémoire. Section Française. Département des Langues Étrangères. Faculté de Lettres et d'Arts. Université de Medan. 2017.

Le but de cette recherche est de savoir les expressions de sentiments qui se trouvent et s'utilisent dans le roman Un Sentiment Plus Fort Que La Peur.

La méthode de la recherche qui est utilisée dans cette recherche est la méthode descriptive qualitative. L'auteur utilise la théorie de Martin et Mabillat et le roman de Marc Levy intitulé Un Sentiment Plus Fort Que La Peur.

Le résultat de cette recherche montre qu'il ya 19 expressions de sentiments utilisées dans ce roman. Ce sont : l'expression de sentiment de la colère, confiance, méfiance, contentement, mécontentement, déception, émotion,ennui, envie, jalousie, espoir, désespoir, gêne, embarras, humeur, indignation, révolte, inquiétude, soulagement, joie, peine, tristesse, peur, plaindre une personne, se plaindre, regret, surprise, et la relation sentimentales. Dans cette analysé, l'expression de contentement et la joie sont plus beaucoup trouvées.
\end{abstract}

Mots Cles : analyse, expression de sentiment, roman

\begin{abstract}
ABSTRAK
Ramawati Lastarida Purba, 2113131036. Analisis ekspresi perasaan dalam novel $U n$ Sentiment Plus Fort Que La Peur. Skripsi. Program Studi Pendidikan Bahasa Perancis. Jurusan Bahasa Asing. Fakultas Bahasa dan Seni. Universitas Negeri Medan. 2017.
\end{abstract}

Tujuan dari penelitian ini adalah untuk mengetahui ekspresi perasaan apa saja yang digunakan didalam novel yang bejudul Un Sentiment Plus Fort Que La Peur

Metode penelitian yang digunakan dalam penelitian ini adalah metode deskriptif kualitatif. Penulis menggunakan teori Martin dan Mabillat dan menggunakan novel karya Marc Levy berjudul Un Sentiment Plus Fort Que La Peur.

Hasil penelitian ini menunjukkan bahwa ada 19 jenis ungkapan perasaan yang digunakan dalam novel ini. Diantaranya adalah: l'expression de sentiment de la colère, confiance, méfiance, contentement, mécontentement, déception, émotion,ennui, envie, jalousie, espoir, désespoir, gêne, embarras, humeur, indignation, révolte, inquiétude, soulagement, joie, 
peine, tristesse, peur, plaindre une personne, se plaindre, regret, surprise, et la relation sentimentales. Dalam analisis ini, ekspresi perasaan la contentement et la joie paling sering digunakan.

\section{Kata Kunci: analisis, ekspresi perasaan, novel}

\section{INTRODUCTION}

La langue est un instrument dont la fonction est pour transmettre quelque chose dans le cœur. À l'autre côté, la langue est un instrument dans le but de faire des interactions ou des communications en disant les pensées, les idées, ou les sentiments. La langue est un système de symbole du son ayant le sens et l'articulation qui est arbitraire et conventionnel, l'utilisée comme un outil de la communication par un groupe d'hommes dans le but d'exprimer les sentiments et les pensées.

En général, la langue se divise en deux, la langue orale et la langue écrite. Ces deux langues sont différentes, la langue orale est la langue qui se produit par l'organe de la parole et elle a besoin d'autres éléments linguistiques, comme des gestes, des mimiques, des regards, et aussi l'intonation de voix pour faire l'interlocuteur comprendre bien l'information que l'on transmet.

Cependant la langue écrite est une langue qu'on transmet un message en symbole arbitraire et ayant la structure de la langue. Alors, quand on écrit un message à quelqu'un, on doit faire attention à la structure, au vocabulaire et aux ponctuations, pour qu'ils soientclaires à comprendre. C'est la raison pour laquelle la langue écrite est plus difficile que celle de l'orale.

Dans la langue orale ou écrite, on emploie des expressions. L'utilisation des expressions a pour but d'assurer les gens avec qui on parle chaque jour. La fonction de l'expression est comme moyen pour persuader quelqu'un sur ce qu'on se sent, car sans expressions, la conversation sera ennuyeuse et on ne peut pas trouver le sentiment avec qui on parle. En français, on trouve fréquemment quelques expressions pour décrire les sentiments, par exemple l'expression de la tristesse, la colère, la surprise, la peur, la joie, etc.

L'émotion peut être d'intensité variable (de très légère à très intense). Les mots que nous utilisons nous permettent de traduire le degré d'intensité (par exemple je suis agaçé, je suis furieux).

La fonction des émotions et des sentiments :Ils nous sont très utiles car ils nous renseignent sur notre état intérieur mais également sur notre environnement.

L'un des medias oùl'on peut trouver les expressions des sentiments est le roman.Le roman est un genre littéraire, caractérisé essentiellement par une narration fictionnelle plus ou moins longue. La place importante faite à l'imagination transparaît dans certaines expressions ou dans certaines acceptations de l'adjectif qui renvoient à l'extraordinaire des personnages, des situations ou de l'intrigue.Le roman est un long récit en prose, qui met en scène des personnages de fiction, engagés dans des aventures imaginaires, parfois présentées comme réelles. C'est aujourd'hui le genre littéraire le plus populaire, si vaste qu'il se subdivise en d'innombrables sous-genres(roman policier, d'aventures, de science-fiction, d'espionnage, d'apprentissage, réaliste, etc.).

Les apprenants de français trouvent les difficultés quand ils aprennent le français surtout les expressions de sentiment à cause du manque de vocabulaire qu'ils doivent utiliser en quelques situations, Par exemple : pour exprimer la colère, habituellement ils utilisent seulement l'expression " être en colère", par exemple "il est en colère " parce que cette expression est la plus couramment utilisée dans le langage courant, bien qu'il y ait beaucoup 
d'autres expressions pour exprimer la colère comme "être vert de rage, être rouge de colère, être dans une colère noire, etc. " Ces expressions sont rarement utilisées parce qu'ils ne les connaissent pas ou bien ils aiment utiliser les expressions fréquentées.

C'est pourquoi, l'auteur s'intéresse à faire une recherche sur les expressions de sentiments pour que les lecteurs aient des connaissances afin d'enrichir l'utilisation des expressions dans les situations précises et plus variées.

L'auteur fait cette recherche en utilisant le roman Un Sentiment Plus Fort Que La Peur, parce que dans ce roman il y a beaucoup d'expressions de sentiments avec une variété de phrases, et aussi les vocabulaires dans ce roman sont faciles à comprendre pour les apprenants de français y compris les débutants. Dans le livre "les Conversations Pratique de l'oral" (2003: 81) Martins et Mabilat affirment qu'il y a plusieurs expressions pour exprimer le sentiment telles que : colère, confiance, méfiance, contentement, mécontentement, déception, émotion,ennui, envie, jalousie, espoir, désespoir, gêne, embarras, humeur, indignation, révolte, inquiétude, soulagement, joie, peine, tristesse, peur, plaindre une personne, se plaindre, regret, surprise.

\section{But de la recherche}

Cette recherché a pour but de savoir les expressions des sentiments qui sont trouvées dans le roman un sentiment plus fort que la peur et savoir l'expression la plus fréquente trouvée dans le roman un sentiment plus fort que la peur.

\section{RECOURS DE THÉORIES}

\section{Analyse} constitués.

Analyse peut devenir le matériau pour faire une recherche tout en ses éléments

SelonDerrida (2002:7) kata analisis berasal dari bahasa Yunani yaitu analyein yang berarti menyelesaikan, menguraikan. C'est-à-dire que le mot «analyse » vient de langue de Grec analyeindont le sens est faire finir, expliquer en détaille. Tandis que selon Rey dans Tarigan $(2012: 11)$ dit que l'analyse est une étude et explication d'un œuvre, d'un texte, d'une situation par la mise en évidence de leurs éléments importants.

L'analyse est une recherche qui expliqe l'objet devient quelque partie pour obtenir un résultat. Paul (2000:93) dit que " l'analyse est une action de décomposer un tout en ses éléments constituants ». Ainsi, l'analyse ne peut pas être séparée d'une recherche parce que l'action de détacher, c'est-à-dire faire la séparation de quelque chose devient des petites parties avec la manière d'identification, de comparaison etc.

Comme cela, on peut conclure que l'analyse est une recherche d'un événement pour connaître la cause. Elle ne peut pas être séparée d'une recherche faite comme toutes les recherches ayant besoin d'analyses de l'auteur en écrivant et créant.

Dans cette recherche, on va faire une analyse sur l'expression. L'expression est une idée fondamentale, ce qui est dit ou peut être dit par le langage, communication des idées ou des sentiments comme la manière de présenter se idées, c'est une chose ce en quoi il y a des rapports qui répondent aux rapports de la chose à exprimer. Et ces expressions sont variées. 


\section{Expression de Sentiment}

Selon Cottraux dans Martrenchard (2003 :30) "l'émotion comme une réponse comportementale et physiologie, brève et intense, qui reflète ou révèle le vécu personel de celui qui est affecté par un événement interne ou externe. Il ajoute que l'émotion est à la fois une sensation et une construction mentale. Il fait également une distinction entre l'émotion qui est brève, ne dure que quelques secondes et renvoie à une intensité et le sentiment qui s'inscrit plus dans la durée".

Ekman dans Jacques Cosnier (2006 :19) dit que "L'expression des émotions de base est reconnue de façon universelle. Quelques nuances apparaissent selon les émotions et les cultures, par exemple la surprise peut être confondue avec la colère.

Alors, l'expression de sentiment est une expression qui indique un sentiment ou de l'état du cœur de quelqu'un à travers son expression de visage et implique les fonctions cognitives du corps, par le biais de la tonalité émise lorsque l'on parle, et à travers les paroles qu'il a prononcées.

Grâce à l'expression, d'autres personnes peuvent connaître et comprendre ce que nous ressentons.

Dans le livre Conversation pratiques de l'oral Martins et Jean Mabilat (2003: 81) il y a quelques expressions pour exprimer des sentiments, ce sont :

\section{La Colère}

Dans le site (http://www.redpsy.com/guide/colere.html), «la colère est une émotion simple qui traduit l'insatisfaction. Elle est vécue à l'égard de ce que nous identifions, à tort ou à raison, comme étant "responsable" de notre frustration. Nous éprouvons donc de la colère envers "l'obstacle" à notre satisfaction ».

Exemples d'expressions de la colère :

Je suis hors de moi, cette expression désigne un état émotionnel particulièrement intense. Par exemple, nous disons que nous sommes hors de nous, lorsque nous éprouvons une violente colère. Ainsi parvenons-nous à figurer un certain manque de maîtrise dans nos réactions lors d'une vive émotion. Des gestes, des paroles, des cris, nous échappent ; nous ne sommes plus maître de nous-mêmes, à tel point parfois que nous ne reconnaissons plus ces actes comme nôtre quand nous les rappelle.

Je haïs mon copain, c'est une expression standard qui marque une réprobation extrême envers une personne, elle s'applique dans les cas de différents affectifs ou dans un cas d'initie profonde. Je haiis est une expression négative mais qui n'est pas forcement impolie, elle exprime juste un rejet violent d'autrui.

\section{La Tristesse}

Dans le site (http://www.cnrtl.fr/definition/tristesse) la tristesse est «un État d'incapacité à éprouver de la joie, à montrer de la gaieté, se traduisant notamment par les traits du visage affaissés, le regard sans éclat.Synonyme. Abattement, affliction, amertume, cafard ${ }^{1}$ (familier), chagrin $^{2}$, dépression, ennui, mélancolie, morosité, nostalgie, peine ».

Exemples d'expressions de la tristesse

Elle est triste, cette expressionindique la personne ressent réellement l'émotion de tristesse « il y a une raison a cette tristesse, cette phrase est polie, elle peut adresser à toute personnes, aux amis, aux personnes àgées.

J'ai de la peine, c'estune expression signifiant que la personne ressent du chagrin, ressent une certaine souffrance psychologique, elle est dans un état de tristesse, d'abattement, nous pouvons avoir de la peine de chose ou pour quelqu'un, nous pouvons aussi avoir de la peine.Et cette phrase est polie, elle peut adresser à toutes personnes, des amis, aux personnes àgées. 


\section{La Joie}

Le site (http://www.cnrtl.fr/definition/joie) affirme que «émotion vive, agréable, limitée dans le temps; sentiment de plénitude qui affecte l'être entier au moment où ses aspirations, ses ambitions, ses désirs ou ses rêves viennent à être satisfaits d'une manière effective ou imaginaire $\gg$.

Exemples d'expressions de la joie

C'est génial, c'est une expression traduisant une satisfaction intense, une approbation total a une situation que l'on trouve excellente et très positive, cette phrase est polie, elle peut adresser à toute personne : des amis, aux personnes àgées.

J'ai le grand plaisir de ma nouvelle maison,c'estune expression de politesse, elle traduit une émotion de fierté, on a grand plaisir a présenter une personne que l'on aime ou a faire quelque chose que l'on aime, c ;est une expression de joie intense. Elle peut adresser à toute personne : des amis, aux personnes àgées.

\section{La Jalousie}

La jalousie est une émotion empreinte d'agressivité envers une personne dont on se figure, à tort ou à raison, qu'elle possède quelque chose que l'on n'a pas et que l'on désire.

Exemples d'expressions de la jalousie :

J'en bave d'envie, c'est une expression d'émotion de convoitise et d'appétit, lorsque l'on désire fortement quelque chose on peut comparer ce désir a de l'appétit pour un plat très alléchant. Baver d'envie n'est pas une expression polie, elle est assez vulgaire, seuls les jeunes gens l'utilisent.

J'en ai envie, c'est une expression plus douce, c'est un désir plus léger, ce n'est pas de la convoitise juste un désir d'avoir. Cette phrase est standard, elle peut adresser à toutes personnes, des amis, aux personnes àgées.

\section{La Peur}

Selon le site (http://www.cnrtl.fr/lexicographie/peur) c'est « un état affectif plus ou moins durable, pouvant débuter par un choc émotif, fait d'appréhension (pouvant aller jusqu'à l'angoisse) et de trouble (pouvant se manifester physiquement par la pâleur, le tremblement, la paralysie, une activité désordonnée notamment), qui accompagne la prise de conscience ou la représentation d'une menace ou d'un danger réel ou imaginaire.Synonyme. Crainte, effroi, épouvante, frayeur, terreur;frousse, pétoche, trouille, venette ».

Exemples d'expressions de la peur:

J'ai les jetons, c'est une expression très familière, elle s'applique a un individu lorsqu'il pense qu'il n'est pas capable de réaliser quelque chose et en éprouve de la peur. Avoir les jetons est une expression utilisée surtout par les jeunes, elle n'est pas très polie.

J'ai le trac, c'est une expression standard, c'est une émotion ressentie par beaucoup de personnes spécialement les artistes, les chanteurs.

\section{RomanUn Sentiment Plus Fort Que La Peur \\ 1. Définition du Roman}

Le mot roman devient du grec c'est-à-dire novella qui signifie avec nouveau ou nouvelle, c'est-à-dire l'histoire de fiction qui est près de la vie humaine.

Dictionnaire Larousse (2004:74) exprime que « un nouvelle est une composition qui appartient au genre du roman, mais qui s'en distingue par un texte plus court, par la simplicité du sujet et par la sobriété du style et de psychologique» 
Nous pouvons conclure de plusieurs théories ci - dessus que le roman est une œuvre littéraire qui a une forme d'une prose et contient une série d'histoire sur un événement ou l'incident qui est expérimenté par quelqu'un dans son entourage qui fait apparaître de conflit.

\section{Résumé du Roman Un Sentiment Plus Fort Que La Peur}

Dans l'épave d'un avion emprisonné sous les glaces du mont Blanc, Suzie Baker retrouve le document qui pourrait rendre justice à sa famille accusée de haute trahison. Mais cette découverte compromettante réveille les réseaux parallèles des services secrets américains.

Entraîné par l'énigmatique et fascinante Suzie Baker, Andrew Stilman, grand reporter au New York Times, mène une enquête devenue indispensable à la survie de la jeune femme. Traqués, manipulés, Suzie et Andrew devront déjouer pièges et illusions jusqu'à toucher du doigt l'un des secrets les mieux gardés de notre temps.

Des personnages qui vous collent à la peau, un suspense haletant... Avec ce nouveau roman, Marc Levy cisèle une histoire d'une modernité surprenante.

Ce nouveau roman nous plonge un peu dans la continuité de "Si c'était à refaire" puisqu'on y retrouve Andrew Stilman, journaliste du New York Times, déjà protagoniste du livre précédent. Mais on y découvre également un nouveau personnage, Suzie Baker, par qui tout va commencer!

Le premier chapitre s'ouvre justement sur Suzie et son obsession soudaine pour l'alpinisme et surtout du Mont Blanc. Et il se conclut sur la découverte du fameux document cité dans le résumé. Mais cette découverte aura un prix pour le jeune femme. On passe ensuite à Andrew Stilman, avec un passage assez déroutant (je ne vous en dis pas plus pour ne pas casser le suspense) ! Andrew va faire la connaissance de Suzie, qui va l'emmener "malgré elle" dans une enquête dont les risques sont énormes. Va s'ensuivre une sorte de jeu de piste dont on ne connaîtra les véritables enjeux qu'à la toute fin du livre. Entretemps, le suspense est intacte et on ne s'ennuie pas.

Côté personnage, on retrouve donc Andrew Stilman, qui (attention spoiler si vous n'avez pas lu le livre précédent) est sorti de l'hôpital ! Quitté par sa femme, il s'est réfugié chez son meilleur ami Simon (ce cher Simon) et dans l'alcool. Il n'a pas écrit un seul article depuis sa sortie et passe ses journées à la bibliothèque. C'est la qu'il va rencontrer Suzie, une jeune femme dont le passé à été assez douloureux à cause d'un scandale qui a sali sa famille. C'est sauver l'honneur de sa famille qu'elle sollicite Andrew. Suzie est un peu le genre de personne auquel on s'attache ou pas! Mais quand on en apprend plus sur elle, on comprend mieux sa personnalité. Autour de ce binôme vont graviter des personnes assez mystèrieuses dont on ne sait jamais vraiment si elle sont honnête ou pas.

On découvre ce qu'est le sentiment plus fort que la peur à travers ce périple bourrés de pièges, de manipulations, et on ne sait pas à qui faire confiance entre les rencontres qui ne sont pas toujours le fruit du hasard et les personnages jouant un double-jeu. Un petit mot au sujet de la couverture, ne la jugeait pas trop vite car elle prend tout son sens au final.

Les relations entres les personnages, que se soit la véritable amitié entre Simon et Andrew ou la relation un peu ambigu entre ce dernier et Suzie, apporte beaucoup à l'histoire, de l'humour pour la première, et un certain dynamisme pour la seconde. Le seul petit bémol que je peux relever, mais il s'agit d'un point de vue strictement personnel, et le sujet réel de l'enquête. Les enjeux politiques et écologiques soulevés ici peuvent être un peu difficiles à suivre.

En résumé, un 14è roman dans la veine du précédent. J'ai pris un grand plaisir à le lire et j'attendrais le suivant toujours impatiemment. 


\section{MÉTHODOLOGIE DE LA RECHERCHE}

La méthode de recherche est très importante dans une recherche afin que le résultat obtenu soit satisfaisant. Avec la méthode de recherche correcte, les problèmes apparus dans la recherche peuvent être résous et le chercheur peut trouver les variétés, si bien que les données de l'analyse peuvent être bien dirigées. Le processus de recherche qui est orientée va produire l'information et l'analyse de données. Cette information peut être acceptée naturellement par tout le monde.

L'auteur utilise la méthode qualitative. Selon Moleong (2001 : 178) «metodologi kualitatif sebagai prosedur penelitian yang menghasilkan data deskriptif berupa kata-kata tertulis atau lisan dari orang-orang dan perilaku yang dapat diamati ». C'est-à-dire la donnée qualitative est une procédure de recherche qui produit la donnée descriptive et se compose par des mots écrits ou orale des personnes et les attitudes qui peuvent être observées.

Selon Bogdan et Taylor (Margono 2005 : 36), penelitian kualitatif ialah proses penelitian yang menghasilkan data deskriptif berupa kata-kata tertulis atau lisan dari orang-orang yang diamati. Ça veut dire, est un procès observées.

La méthode de cette recherche va expliquer une condition de vérité, et donner la priorité à la donnée que la théorie, où l'auteur va analyser le roman français intitulé un sentiment plus fort que la peur. Avec cette méthode, l'auteur va analyser la structure des expressions de sentiment trouvée dans ce roman connu.

Pour accumuler les données dans cette recherche, on utilise la recherche en bibliothèque en compromettant le roman Français Un Sentiment Plus Fort Que La Peur.

\section{RESULTAT DE LA RECHERCHE}

Après avoir analysé de données, on a obtenu la distribution de l'utilisation des expressions de sentiments dans le roman Un Sentiment Plus Fort Que La Peur.

Tableau 4.1

La récapitulation de l'expressions de sentiments dans le roman un sentiment plus fort que la peur

\begin{tabular}{|l|l|c|c|}
\hline No. & Les expressions de sentiments & Fréquence & Pourcentage $(\%)$ \\
\hline 1. & Colère & 9 & 3,56 \\
\hline 2. & Confiance & 16 & 6,32 \\
& Méfiance & 8 & 3,16 \\
\hline 3. & Contentement & 25 & 9,88 \\
& Mécontentement & 12 & 4,74 \\
\hline 4. & Déception & 7 & 2,76 \\
\hline 5. & Envie & 1 & 0,39 \\
& Jalousie & 2 & 0,79 \\
\hline 6. & Espoir & 23 & 9,09 \\
& Désespoir & 11 & 4,34 \\
\hline 7. & Gêne & 1 & 2,37 \\
& Embarras & 2 & 0,39 \\
\hline 8. & Humeur & & 0,79 \\
\hline
\end{tabular}




\begin{tabular}{|l|l|c|c|}
\hline 9. & Indignation & 8 & 3,16 \\
& Révolte & 7 & 2,76 \\
\hline 10. & Inquiétude & 15 & 5,92 \\
& Soulagement & 8 & 3,16 \\
\hline 11. & Joie & 24 & 9,48 \\
\hline 12. & Peine & 10 & 3,95 \\
& Tristesse & 1 & 1,97 \\
\hline 13. & Peur & 2 & 0,39 \\
\hline 14. & Plaindre une personne & 11 & 0,79 \\
\hline 15. & Se plaindre & 10 & 4,34 \\
\hline 16. & Regret & 9 & 3,95 \\
\hline 17. & Surprise & 16 & 3,55 \\
\hline 18. & Rélations Sentimentales & 4 & 6,32 \\
\hline 19. & Émotion & 253 & 1,58 \\
\hline & Total & & 100 \\
\hline
\end{tabular}

Il y a 19 expressions de sentiment qui se trouvent dans le roman un sentiment plus fort que la peur. Il se trouve 253 expressions de sentiments, ce sont l'expression de la colère est 9 fois $(3.55 \%)$, l'expression de confiance est 16 fois $(6.32 \%)$, l'expression de méfiance est 8 fois $(3.16 \%)$, l'expression de contentement est 25 fois $(9.88 \%)$, l'expression mécontentement est 12 fois $(4.74 \%)$, l'expression de la déception est 7 fois $(2.76 \%)$, l'expression de l'émotion est 4 fois (1.58\%), l'expression de l'envie est 1 fois $(0.39 \%)$, l'expression de la jalousie est 2 fois $(0.79 \%)$, l'expression de l'espoir 23 fois $(9.09 \%)$, l'expression de désespoir est 11 fois (4.34\%), l'expression de la gêne est 6 fois $(2.37 \%)$, l'expression d'embarras est 1 fois $(0.39 \%)$, l'expression de l'humeur est 2 fois $(0.79 \%)$, l'expression de l'indignation est 8 fois (3.16\%), l'expression de révolte est 7 fois $(2.76 \%)$, l'expression de l'inquiétude est 15 fois (5.92\%), l'expression de soulagement est 8 fois $(3.16 \%)$, l'expression de la joie est 24 fois (9.48\%), l'expression de la peine est 10 fois (3.95\%), l'expression de la tristesse 5 fois $(1.97 \%)$, l'expression de la peur est 1 fois $(0.39 \%)$, l'expression de plaindre une personne est 2 fois $(0.79 \%)$, l'expression de se plaindre est 11 fois (4.34\%), l'expression de la regret est 10 fois $(3.95 \%)$, l'expression de la surprise est 9 fois $(3.55 \%)$, et l'expression de la relations sentimentales est 16 fois $(6.32 \%)$.

Tableau 4.2. Les expressions de sentiments trouvées dans le roman un sentiment plus fort que la peur chapitre 1

\begin{tabular}{|l|l|c|c|}
\hline No. & Les expressions de sentiments & Fréquence & Pourcentage(\%) \\
\hline 1. & Colère & 3 & 10 \\
\hline 2. & Confiance & 2 & 6,66 \\
& Méfiance & 1 & 3,33 \\
\hline 3. & Contentement & 3 & 10 \\
& Mécontentement & 3 & 10 \\
\hline 4. & Déception & 1 & 3,33 \\
\hline 5. & Espoir & 3 & 10 \\
& Désespoir & 2 & 6,66 \\
\hline 6. & Indignation & 1 & 3,33 \\
\hline
\end{tabular}




\begin{tabular}{|l|l|c|c|}
\hline 7. & Inquiétude & 1 & 3,33 \\
& Soulagement & 1 & 3,33 \\
\hline 8. & Joie & 4 & 13,3 \\
\hline 9. & Peine & 1 & 3,33 \\
\hline 10. & Se plaindre & 2 & 6,66 \\
\hline 11. & Regret & 1 & 3,33 \\
\hline 12. & Surprise & 30 & 3,33 \\
\hline & Total & 100 \\
\hline
\end{tabular}

Dans le chapitre I, il se trouve 30 expressions de sentiments. L'expression de sentiments de la colère est 3 fois $(10 \%)$, l'expression de sentiments de la confiance est 2 fois $(6,66 \%)$, méfiance est 1 fois $(3,33 \%)$, contentement est 3 fois $(10 \%)$, mécontentement est 3 fois $(10 \%)$, déception est 1 fois $(3,33 \%)$, espoir est 3 fois (10\%), désespoir est 2 fois $(6,66 \%), 1$ 'indignation/révolte est 1 fois $(3,33 \%)$, l'inquiétude est 1 fois $(3,33 \%)$, soulagement est 1 fois $(3,33 \%)$, la joie est 4 fois $(13,3 \%)$, peine/tristesse est 1 fois $(3,33 \%)$, se plaindre est 2 fois $(6,66 \%)$, regret est 1 fois $(3,33 \%)$, et la surprise est 1 fois $(3,33 \%)$.

L'expression de sentiment de la joie est plus fréquente trouvée dans le chapitre 1. Parce que dans ce chapitre, il parle que Suzie retrouve le document qui pourrait rendre justice à sa famille accusée de haute trahison.

Les expressions du sentiment dans le chapitre I :

1. Les expressions de sentiments de la Colère

a) Tu renonces jamais quand tu as une idée en tête?

(Cette parole est une expression de la colère. Prononcée par Shamir. Il est en colère quand Suzie lui demande d'utiliser la phrase tu renonces jamais quand tu as une idée en tête?).

b) L'accompagner le mettait en colére

(Cette parole est une expression de la colère. On peut voir la phrase utilise sujet + en colère ).

c) Vous ne me connaissez pas

(Cette parole est une expression de la colère. Prononcée par Suzie.)

2. L'expression de sentiments de la Confiance/Méfiance

a) Je me suis toujours fiée à mon instinct.

(Cette parole est une expression de la confiance. Parce'que dans cette phrase il y a une grande confiace à d'eux-mêmes sur quelque'chose).

b) D'accord.

(Cette parole est une expression de la confiance)

c) Il ne fallait pas cédér à la peur. 
(Cette parole est une expression de la confiance. On peut voir du sens de cette phrase ).

3. L'expression de sentiments de la Contentement/Mécontentement

a) C'est précisément.

(Cette parole est une expression de la contentement. Prononcée par Shamir quand il veut parler à Suzie. Il se sent content parce que Suzie lui répond.)

b) Merci du compliment

(Cette parole est une expression de la contentement. Parce'qu'un personage se sent très bien quand elle reoit un compliment).

c) C'etait hors de question.

(Cette parole est une expression de la mécontentement.)

d) Tu as une meilleure idée?

(Cette parole est une expression de la mécontentement. Prononcée par Shamir pour demander Suzie, parce'qu'elle demande quelque chose qui ne pas important.)

4. L'expression de sentiments de la Déception

a) Les deux ne sont pas incompatibles.

(Cette parole est une expression de la déception. Prononcée par Suzie. Dans cette phrase il utilise le mot incompatibles, ce qui signifie qu'il ya la déception en raison de la non-concordance).

b) Tu n'es pas prête.

(Cette parole est une expression de la déception, on sait que cela est une expression de la déception c'est de la signification d'une phrase est utilisée.)

5. L'expression de sentiments de l'Espoir/Désespoir

a) J'aimerais ne pas..

(Cette parole est une expression de la désespoir. On peut voir, il utilise le mot futur qui disent qu'il ya de l'espoir en Shamir).

b) J'espère que vous..

(Cette parole est une expression de l'espoir. On peut voir, il utilise le mot futur qui disent qu'il ya de l'espoir en Shamir).

6. L'expression de sentiments de l'Indignation/Révolte

a) Pas comme ça.. 
(Cette parole est une expression de la révolte. Prononcée par Shamir. il n'est pas d'accord avec ce qui a été dit par Suzie, donc il est révolté).

7. L'expression de sentiments de l'Inquiétude/Soulagement

a) Il fallait être calme.

(Cette parole est une expression de l'inquiétude. Il utilise le phrase être + calme. Ici, on peut voir il ya quelqu'un a essayé de calmer son ami qui était inquiet de quelque chose.)

8. L'expression de sentiments de la Joie

a) Les gens souriants

(Cette parole est une expression de la joie. On peut voir le mot sourire qu'utilise dans cette phrase qui explique qu'il ya une joie qui les rendait joyeux)

b) C'est extatique.

(Cette parole est une expression de la joie, prononcée par Suzie. Dans cette phrase il utilise le mot extatique pour exprimer sa joie.)

c) Suzie avait été heureuse que.. (page 22)

(Cette parole est une expression de la joie. Suzie se sens très heureuse quand elle a été invité pour à dîner ensemble avec Shamir. Cette phrase utiliser le mot heureuse)

d) C'était exactement.

(Cette parole est une expression de la joie. Il utilise le mot exactement pour exprimer la joie)

9. L'expression de sentiments de la Peine/tristesse

a) Elle avait cru.

(Cette parole est une expression de la peine. Suzie avait de la difficulté face à sa première leçon, ce qu'elle pensait. Elle utilise le mot cru.)

10. L'expression de sentiments de Se plaindre

a) Je ne suis pas une meilleure alphiniste.

(Cette parole est une expression de se plaindre. Cette phrase prononcée par Suzie, quand Shamir dit qu'il a besoin d'un guide et d'un professeur patient, mais Suzie pense qu'elle n'est pas une meilleure alphiniste.)

b) C'est ne pas plus important.

(Cette parole est une expression de se plaindre )

11. L'expression de sentiments de la Regret

a) Je te le promets. 
(Cette parole est une expression de la regret. Prononcée par Shamir. Dans cette phrase il utilise le mot promet ce qui signifie la regret.)

b) Malhonnête!

(Cette parole est une expression de la regret. Prononcée par Suzie. Elle a dit qu'elle a une proposition malhonnête à Shamir faire.)

12. L'expression de sentiments de la Surprise

\section{a) Pourquoi lui ?}

(Cette parole est une expression de la surprise. Prononcée par Shamir.)

\section{Conclusion}

En se fondant sur les résultats de la recherche présentées dans le chapitre IV, l'auteur peut tirer la conclusion comme suivante :

1. Il y a 19 expressions du sentiment qui se trouvent dans le roman un sentiment plus fort que la peur. Il se trouve 253 expressions de sentiments, ce sont l'expression de la colère étant 9 fois $(3.55 \%)$, l'expression de confiance étant 16 fois $(6.32 \%)$, l'expression de méfiance étant 8 fois $(3.16 \%)$, l'expression de contentement étant 25 fois $(9.88 \%)$, l'expression mécontentement étant 12 fois (4.74\%), l'expression de la déception étant 7 fois (2.76\%), l'expression de l'émotion étant 4 fois (1.58\%), l'expression de l'envie étant 1 fois $(0.39 \%)$, l'expression de la jalousie étant 2 fois $(0.79 \%)$, l'expression de l'espoir étant 23 fois $(9.09 \%)$, l'expression de désespoir étant 11 fois $(4.34 \%)$, l'expression de la gêne étant 6 fois (2.37\%), l'expression d'embarras étant 1 fois $(0.39 \%)$, l'expression de l'humeur étant 2 fois $(0.79 \%)$, l'expression de l'indignation étant 8 fois $(3.16 \%)$, l'expression de révolte étant 7 fois $(2.76 \%)$, l'expression de l'inquiétude étant 15 fois $(5.92 \%)$, l'expression de soulagement étant 8 fois (3.16\%), l'expression de la joie étant 24 fois $(9.48 \%)$, l'expression de la peine étant 10 fois (3.95\%), l'expression de la tristesse étant 5 fois (1.97\%), l'expression de la peur étant 1 fois $(0.39 \%)$, l'expression de plaindre une personne étant 2 fois $(0.79 \%)$, l'expression de se plaindre étant11 fois $(4.34 \%)$, l'expression de la regret étant 10 fois $(3.95 \%)$, l'expression de la surprise étant 9 fois $(3.55 \%)$, et l'expression de la relation sentimentale étant 16 fois $(6.32 \%)$.

3. L'expression de la contentement et la joie sont plus fréquents trouvées. Parce qu'une partie de ce roman parle de l'histoire d'amour entre Suzie Baker et son avocat nommé Andrew. Beaucoup d'expressions de la joie qu'ils ressentent, et de plus, finallement Suzie a trouvé le document secret qui peut aider sa famille à être sauvé du judge de l'État. Dans ce roman, on découvre ce que c'est le sentiment plus fort que la peur, parce qu'il parle tant de tricher, manipulations, entensiblement des gens qui prennent un double-jeu.Et puis, il raconte aussi la vie étant plein de problèmes très compliqués et imprévus. Donc, on ne doit pas apprécier quelqu'un de son apperence.

\section{BIBLIOGRAPHIE}

Abbadie, Christian, 1998. L'expression française. Paris : Presses Universitaires de Grenoble.

Ahmadi, A. 1998. PsikologiUmum, Jakarta: PT.RinekaCipta. 
Arikunto, Suharsimi. 2007. Manajemen Penelitian. Jakarta. Rineka Cipta.

Barféty, Michèle. 2005. Expression Orale «Niveau 3 ». Paris : CLE

International.

Bercherelle. 1990. La Grammaire Pour Tous. Paris :Hatier.

Hubere, A et Ruyon, R P. 1984. Psychologi of Adjustement.Homewood : The DorseyPress.

Larivey, Michelle. 2000. Guide des Émotions. Paris. Ressources en

Développement inc.

Larousse, 2004. Dictionnaire de français. Paris : Bordas.

Margono, 2005. Metodologi Penelitian Pendidikan. Jakarta : Rineka Cipta.

Martin et Mabillat. 2003. Conversation Pratiques de L'Oral. Paris. Les Éditions

Didier.

Moleong, 2001. Metodologi Penelitian Kualitatif. Bandung : Remaja Rosdakarya.

Muhammad. 2011. Metode Penelitian Bahasa. Jogjakarta. Ar-ruzz MEDIA.

Robert, Jean-Pierre. 2002. Dictionnaire Pratique de Didactique. Paris : OPHRYS

Tarigan, EndaMorina. 2012. L'analyse de l'Identification du Vocabulaire des

Emotions Dans Le Roman 'Tristan et Iseut'. Mémoire. Medan. UNIMED Press.

Tarigan, Jantriana. 2015. Analyse de l'Expression de Sentiment dans le Film

"Comme Un Chef"

Widodo, Bambang. 2008. Metode Penelitian. Jakarta. PTIK.Press.

\section{SITOGRAPHIE}

https://gaelleana.blogspot.co.id/2014/07/exprimer-les-sentiments-et-les-

emotions.html (accès le 05 juin 2016)

http://www.francaisfacile.com/exercices/exercice-francais-2/exercice-francais89484.php (accès 29 août 2016)

http://www.redpsy.com/guide/colere.html (accès 15 juillet 2016)

http://seliberer.fr/le-vocabulaire-des-emotions-et-sentiment(accès 31 mai 2017) 